Research Paper

\title{
Ginsenoside Rgl alleviates acute liver injury through the induction of autophagy and suppressing NF-KB/NLRP3 inflammasome signaling pathway
}

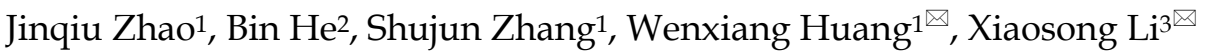 \\ 1. Department of Infectious Diseases, The First Affiliated Hospital of Chongqing Medical University, Chongqing, 400016, China. \\ 2. Department of Orthopedics, The First Affiliated Hospital of Chongqing Medical University, Chongqing, 400016, China. \\ 3. Clinical Molecular Medicine Testing Center, The First Affiliated Hospital of Chongqing Medical University, Chongqing, 400016, China. \\ $\triangle$ Corresponding authors: Wenxiang Huang, wenxiang_huang@163.com. Xiaosong Li, Email: lixiaosong@cqmu.edu.cn, Telephone: 008602389011210, Fax \\ number: 008602389011210. Postal address (all authors): No. 1 Yi Xue Yuan Road, Yuzhong District, Chongqing, 400016, China.
}

(c) The author(s). This is an open access article distributed under the terms of the Creative Commons Attribution License (https://creativecommons.org/licenses/by/4.0/). See http://ivyspring.com/terms for full terms and conditions.

Received: 2020.07.21; Accepted: 2020.12.02; Published: 2021.01.23

\begin{abstract}
Background: Severe hepatitis is a common cause of chronic or acute liver disease and autophagy might play an important role in cellular response to inflammation and injury. It has been reported that Ginsenoside-Rgl (G-Rgl) has strong hepatoprotective effects for acute liver injury, but its protective mechanisms have not yet been elucidated. This study aims to explore the detailed molecular mechanisms of G-Rgl on acute liver injury via autophagy.

Methods: The role of G-Rgl by autophagic induction was studied in the mouse model of acute liver injury which induced by carbon tetrachloride $(\mathrm{CCl} 4)$. Liver function, inflammatory reaction and apoptosis were detected when autophagy has been inhibited by 3-MA or stimulated by RPA. MCC 950 and ATP were applied to investigate the role of NLRP3 inflammasome in acute liver injury. The differential expression of NF-KB, NLRP3 inflammasome, caspase 1, caspase 3, IL-1B, IL-18, LC3-I, LC3-II, Beclin-1, PINK 1 and Parkin have been detected by the quantitative real-time polymerase chain reaction (qRT-PCR) and Western blot.

Results: G-Rgl could decrease ALT, AST, TNF- $\alpha$, IL-1 $\beta$ and IL- 6 in mice with CCl4-induced acute liver injury. The change of autophagy and apoptosis after the treatment of 3-MA or RPA demonstrated that the autophagy played a key role in the protective effect of $\mathrm{G}-\mathrm{Rg} 1$ in acute liver injury. The enhancement of G-Rgl promoted-autophagy resulted in the significant decrease in NF-KB, NLRP3 inflammasome, caspase 1 , caspase $3, I L-1 \beta$ and IL-18, which suggesting that NF-KB/NLRP3 inflammasome signaling pathway was associated with the autophagy induced by $\mathrm{G}-\mathrm{Rg} 1$ in acute liver injury.

Conclusion: G-Rgl ameliorated acute liver injury via the autophagy, which may be related to NF-KB/NLRP3 inflammasome signaling pathway.
\end{abstract}

Key words: Ginsenoside-Rg1, acute liver injury, autophagy, NF-kB, NLRP3 inflammasome.

\section{Introduction}

Acute liver injury can result in liver function abnormality, and is caused by many reasons including viral infection, abuse of drugs or alcohol, ingestion of toxic substance etc [1-3]. Serious liver injury may lead to liver failure or death $[4,5]$. Acute liver failure is an inflammation-mediated hepatocellular injury process, during which hepatocellular apoptosis and hemorrhagic will necrosis occur $[6,7]$. The prognosis for acute liver failure is extremely poor, and only liver transplantation may be effective to treat the end stage of acute liver failure [8].

Ginsenoside Rg1 (G-Rg1) is the most abundant ingredient of Panax ginseng, and exerts strong influence on reactive oxygen stress, angiogenesis, neuronal differentiation, and anti-inflammation 
[9-12]. Previous studies have explored that the hepatoprotective effects of G-Rg1, and demonstrated the potential of G-Rg1 in reducing the production of proinflammatory factors such as TNF-a [13] and alleviating liver fibrosis [14]. Our previous studies have found that G-Rg1 had the ability to improve liver function and inhibit caspase-dependent hepatocellular apoptosis in mouse model of acute liver failure [15].

Autophagy, a highly conserved catabolic process could prevent cell damage and promote survival in the event of energy or nutrient shortage and respond to various cytotoxic insults [16-18]. It had multiple functions in cell survival, proliferation and apoptosis in mammals $[19,20]$. Some studies have reported that autophagy consist of several procedures including the sequestration of a region of cytosol in double-membrane compartments, the formation of autophagosomes, the fusion of autophagosomes with lysosomes and degradation dependent on lysosome $[21,22]$.

Given the above information, we proposed that G-Rg1 might play a protective role in acute liver injury by regulating autophagic pathway. In the mice model of carbon tetrachloride ( $\mathrm{CCl} 4)$-induced acute liver injury, the liver function, inflammatory reaction and apoptosis were analyzed after the treatment of 3-methyladenine (3-MA) or enhanced rapamycin (RPA) in order to explore the role of autophagy in the protective effect of G-Rg1. Then qRT-PCR and Western blot were conducted to detect NF-kB, NLRP3 inflammasome and autophagic proteins for investigating the relationship between NF-kB/NLRP3 inflammasome signaling pathway and autophagy.

\section{Results}

\section{G-Rgl had strong hepatoprotective effects in mice with $\mathrm{CCl} 4$-induced acute liver injury}

To investigate the role of G-Rg1 in acute liver injury, the serum alanine aminotransferase (ALT) and aspartate aminotransferase (AST), TNF- $\alpha$, IL-1 $\beta$, IL-6 of mice were detected after $24 \mathrm{~h}$ injection with $\mathrm{CCl} 4$ (Figure 1A). The levels of ALT, AST, TNF- $\alpha$, IL-1 $\beta$ and IL-6 in G-Rg1 treatment group were significantly reduced than those in control group, indicating that G-Rg1 treatment had the ability to improve liver function and inhibit inflammatory reaction. In addition, the autophagy agonist RPA in combination with G-Rg1 was associated with substantially reduced ALT, AST, TNF- $\alpha$, IL-1 $\beta$ and IL-6 than G-Rg1 treatment group. Consistently, the autophagy inhibitor 3-MA addition resulted in the increase in ALT, AST, TNF- $\alpha$, IL-1 $\beta$ and IL-6 than G-Rg1 treatment group. The HE staining also showed that G-Rg1 had strong hepatoprotective effects in mice with CCl4-induced acute liver injury (Figure 1B). The above results suggested that autophagy may play an important role in the protective effect of G-Rg1 in acute liver injury.

\section{G-Rg 1 could promote hepatocyte autophagy in mice with CCl4-induced acute liver injury}

In order to explore the mechanisms of G-Rg1 on acute liver injury in this study, then, the further research on protective effect of G-Rg1 for acute liver injury have been implemented. G-Rg1 could regulate autophagy in mice that had been exposed to $\mathrm{CCl} 4$ for $24 \mathrm{~h}$ (Figure 2). Flow cytometry showed that G-Rg1 could promote autophagy significantly than the

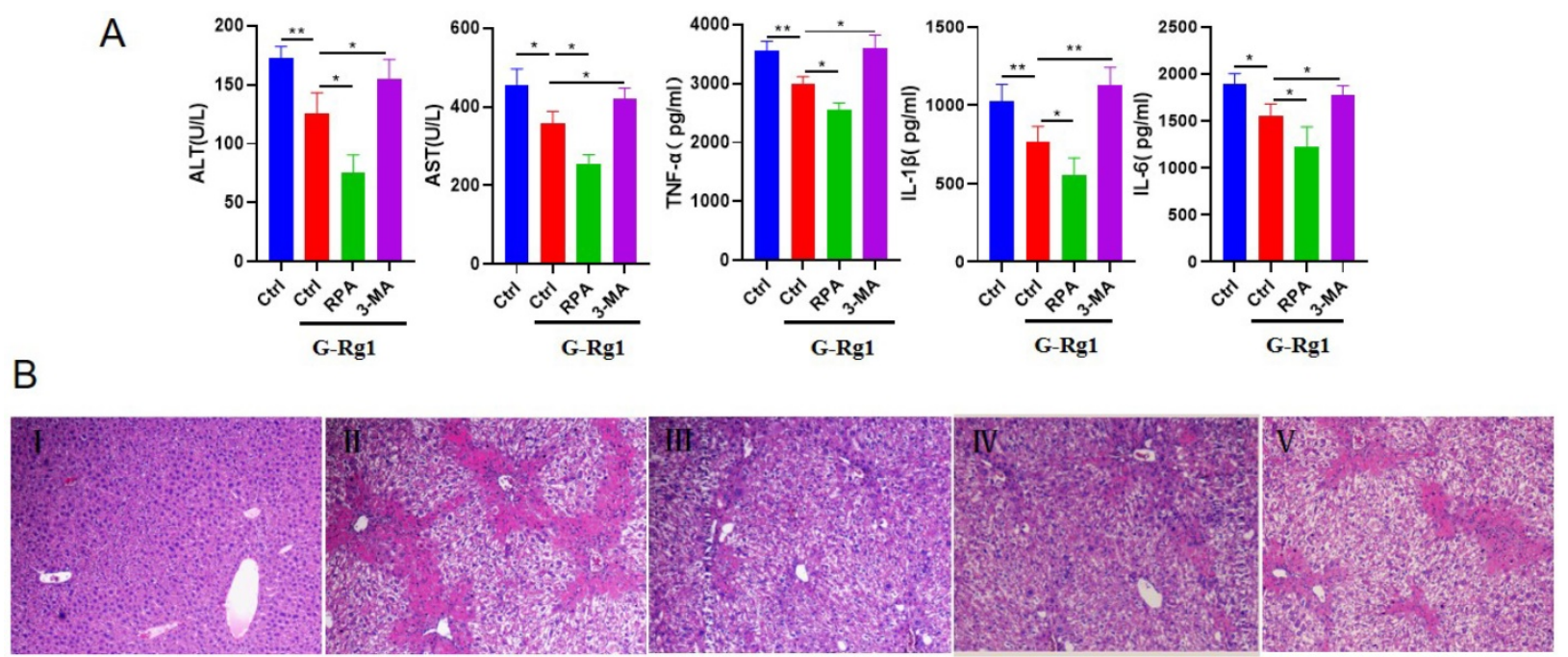

Figure 1. G-Rgl significantly improved hepatic function and inhibited inflammatory reaction in mice with CCl 4 -induced acute liver injury. A: Serum AST, ALT, TNF- $\alpha$, IL-1 $\beta$, IL-6 were measured in control group, G-Rgl $(4 \mathrm{mg} / \mathrm{ml})$ control group, G-Rgl $(4 \mathrm{mg} / \mathrm{ml})+\mathrm{RPA}(1 \mathrm{mg} / \mathrm{kg}) \mathrm{group}$ and G-Rgl $(4 \mathrm{mg} / \mathrm{ml})+3-\mathrm{MA}(1 \mathrm{mg} / \mathrm{kg}) \mathrm{group}$ have been implemented after $24 \mathrm{~h}$ of $\mathrm{CCl}_{4}$ injection $(\mathrm{n}=3)$. $* \mathrm{P}<0.05$, $* * \mathrm{P}<0.01$. B: HE staining, I : normal liver tissue, II: CCL4 group, III: G-Rg1 group, IV: G-Rg1+RPA (autophagy agonist) group, V: G-Rgl+3-MA (autophagy inhibitor) group. 
A

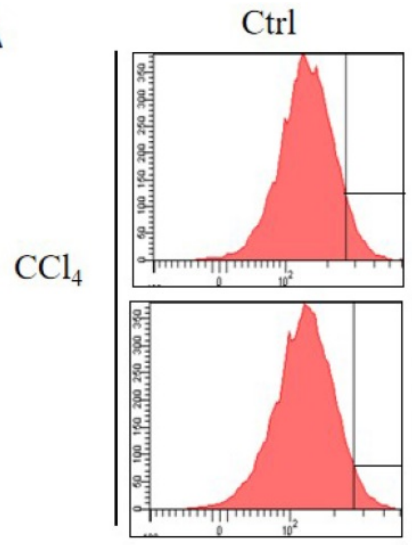

3-MA+G-Rg1

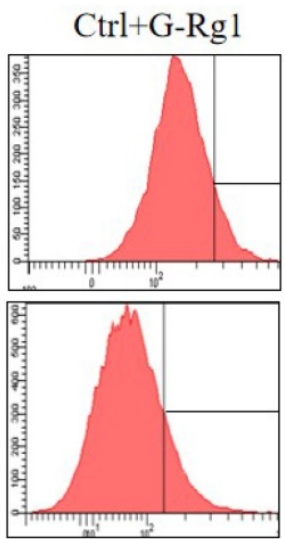

$\mathrm{RPA}+\mathrm{G}-\mathrm{Rg} 1$
B

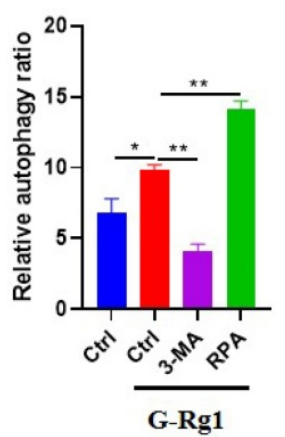

Figure 2. G-Rg1 promoted autophagy for acute liver injury in vivo. The cells were subjected to flow cytometry for autophagy detection after $24 \mathrm{~h}$ of treatment in control group, G-Rgl $(4 \mathrm{mg} / \mathrm{ml})$ control group, G-Rgl $(4 \mathrm{mg} / \mathrm{ml})+3-\mathrm{MA}(1 \mathrm{mg} / \mathrm{kg})$ group and G-Rgl $(4 \mathrm{mg} / \mathrm{ml})+\mathrm{RPA}(1 \mathrm{mg} / \mathrm{kg}) \operatorname{group}(\mathrm{n}=3)$. serum ALT, AST, TNF-a, IL-1 $\beta$ and IL-6 were examined at $24 \mathrm{~h}$ after the $\mathrm{CCl} 4$ injection. When compared to the control intervention, the autophagy agonist RPA could reduce the levels of ALT, AST, TNF- $\alpha$, IL-1 $\beta$ and IL-6, while the autophagy inhibitor 3-MA showed significantly higher ALT, AST, TNF- $\alpha$, IL-1 $\beta$ and IL-6 than autophagy agonist RPA (Figure 4A). These results suggested that the induction of autophagy improved liver function and inhibited inflammatory reaction in CCl4-induced acute liver injury. In addition, the NLRP3 inflammasome inhibitor MCC950 could reduce the levels of ALT, AST, IL-1 $\beta$ and IL- 6 than the control intervention, but had no obvious effect on TNF-a. The NLRP3 inflammasome agonist ATP could increase the levels of ALT, AST, IL-1 $\beta$ and IL-6 than the NLRP3 inflammasome inhibitor MCC950, but there was no statistical difference in TNF-a between two groups (Figure 4B). Above studies implied that NLRP3 inflammasome activation might aggravate $\mathrm{CCl}$-induced acute liver injury.

\section{G-Rgl promoted autophagy through the NF-KB/NLRP3 inflammasome signaling pathway}

Our results revealed that NLRP3 inflammasome significantly increased the inflammatory reactions and reduced liver function in mice with CCl4-induced acute liver injury. Our previous studies have reported that G-Rg1 had the capability to decrease the expression of NF-kB in acute liver injury. G-Rg1 treatment group could reduce the expression of NF-KB, NLRP3 inflammasome, caspase 1, caspase 3, IL-1 $\beta$ and IL-18, but increase the level of LC3-II, PINK1 and Parkin than the control group. The results were consistent with those when comparing G-Rg1 plus autophagy agonist RPA with G-Rg1 treatment group, and comparing G-Rg1 treatment group with G-Rg1 plus autophagy inhibitor 3-MA (Figure 5A). These outcomes were also confirmed by Western blot in terms of NF-KB, NLRP3 inflammasome, caspase 1, caspase 3, IL-1 $\beta$, IL-18, LC3-I, LC3-II, PINK1 and Parkin in each group (Figure 5B). In addition, G-Rg1 plus autophagy agonist RPA showed higer levels of Beclin-1 than G-Rg1 plus autophagy inhibitor 3-MA, in mice with $\mathrm{CCl} 4$-induced acute liver injury. The 
which was consistent with that when comparing G-Rg1 with G-Rg1 plus autophagy inhibitor 3-MA (Figure 5). These results indicated that G-Rg1triggered autophagy could result in the reduction of NF-KB and NLRP3 inflammasome and the inhibition of G-Rg1-triggered autophagy was associated with the increase in NF-KB and NLRP3 inflammasome, which suggesting that NF-kB/NLRP3 inflammasome signaling pathway might participate in the G-Rg1-promoted autophagy in mice with CCl4-induced acute liver injury.

A
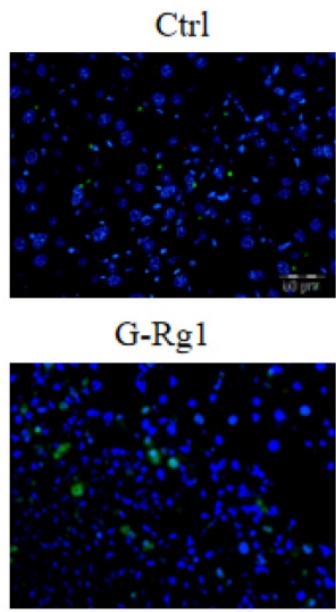

G-Rg1
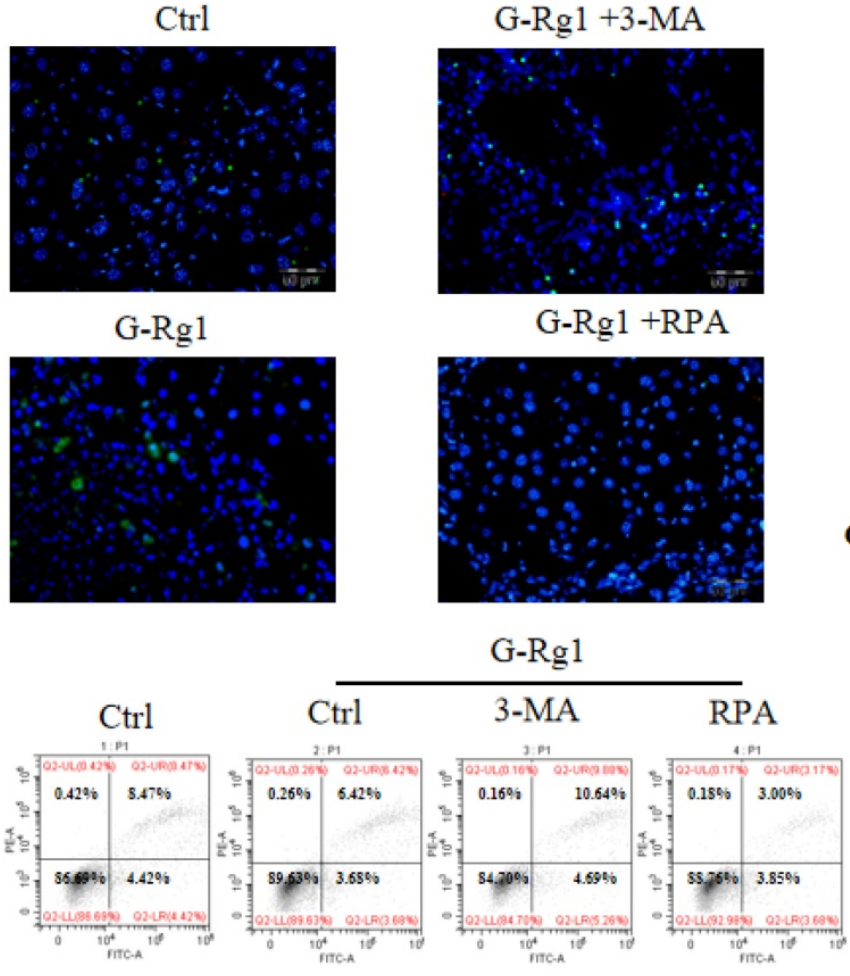

G-Rg1

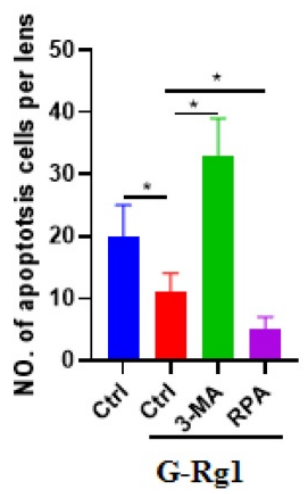

G-Rgl

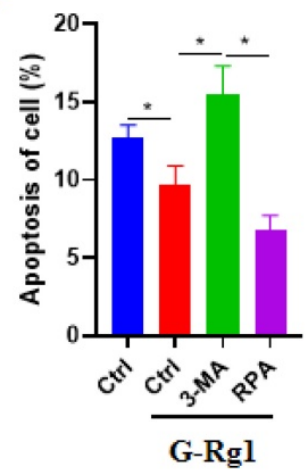

Figure 3. G-Rgl suppressed apoptosis in mice with $\mathbf{C C l}_{4}$-induced acute liver injury through autophagic induction. (A) Apoptotic cells were stained with a TUNEL apoptosis detection kit in control group, G-Rgl $(4 \mathrm{mg} / \mathrm{ml})$ control group, G-Rgl $(4 \mathrm{mg} / \mathrm{ml})+\mathrm{RPA}(1 \mathrm{mg} / \mathrm{kg}) \mathrm{group}$ and G-Rgl(4 mg/ml) $+3-\mathrm{MA}(1 \mathrm{mg} / \mathrm{kg}) \mathrm{group}$ at $24 \mathrm{~h}$ after the $\mathrm{CCl}_{4}$ injection. All of the images were obtained on an inverted fluorescence microscope. (B) Flow cytometry was also performed to detect the apoptotic hepatocytes ( $\mathrm{n}=3$ ). $* \mathrm{P}<0.05$.
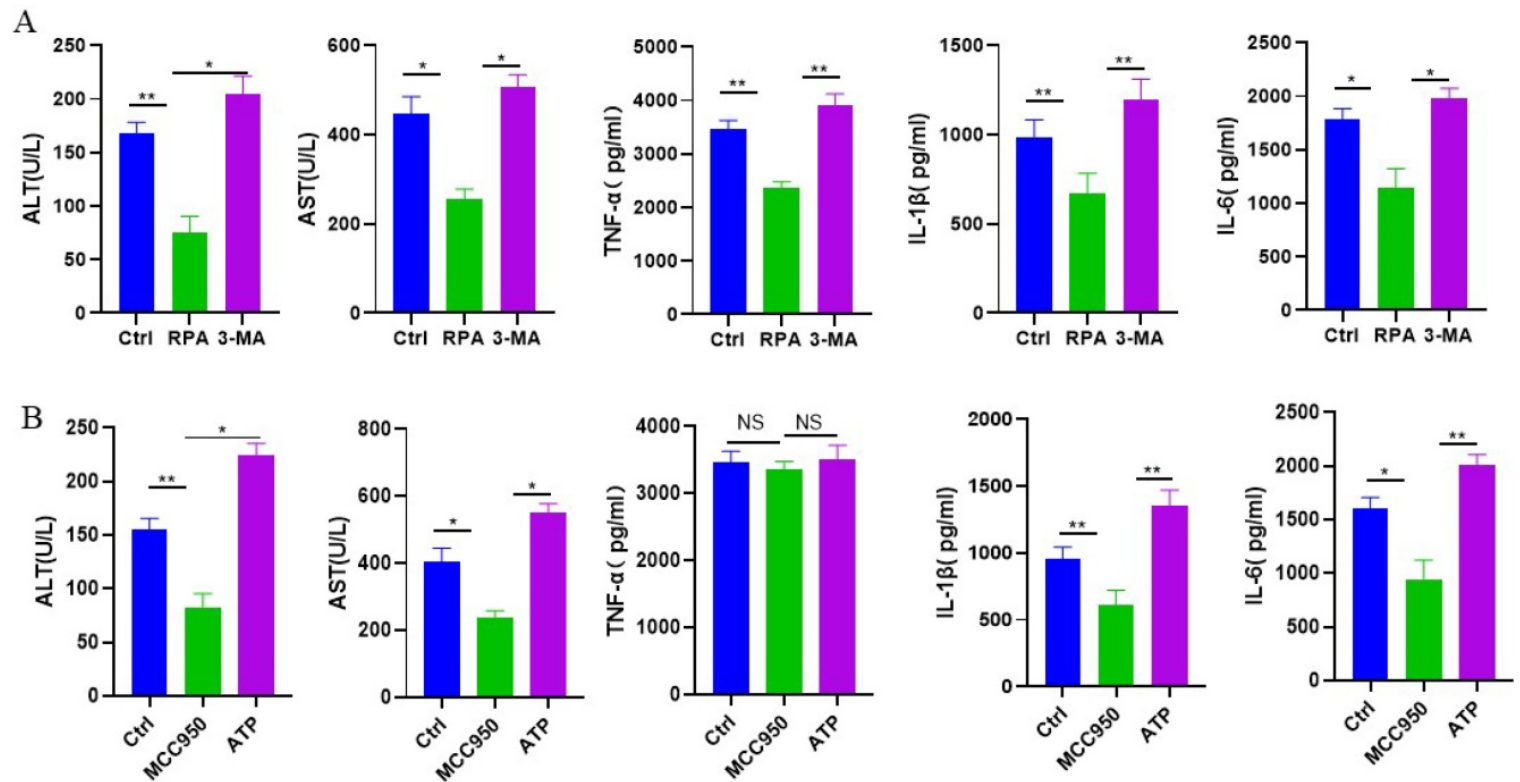

Figure 4. The roles of autophagy and NLRP3 inflammasome in mice with $\mathbf{C C l}_{4}$-induced acute liver injury. (A) Serum AST, ALT, TNF- $\alpha$, IL- $1 \beta$, IL- 6 were measured in control group, RPA group and 3-MA group $24 \mathrm{~h}$ after the CCl 4 injection. (B) Serum AST, ALT, TNF- $\alpha$, IL- $1 \beta$, IL-6 were measured in control group, MCC950 group and ATP group $24 \mathrm{~h}$ after the $\mathrm{CCl}_{4}$ injection $(\mathrm{n}=3)$. $* \mathrm{P}<0.05$, $* * \mathrm{P}<0.01, \mathrm{NS}$, no significant. 
A
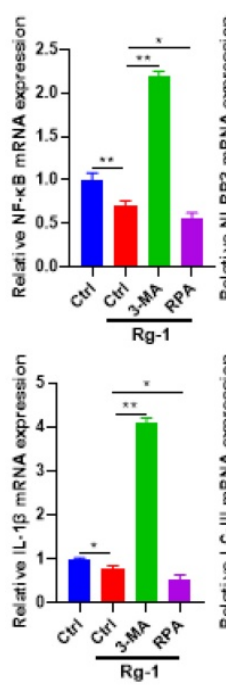
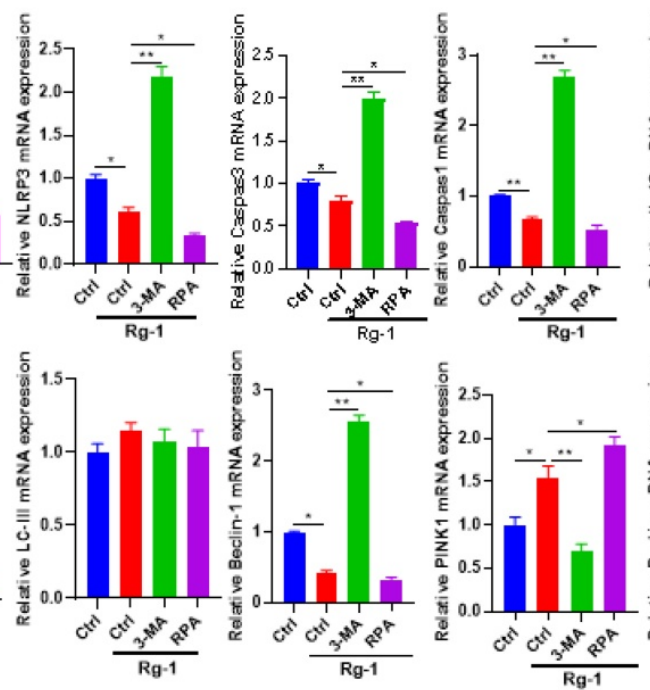
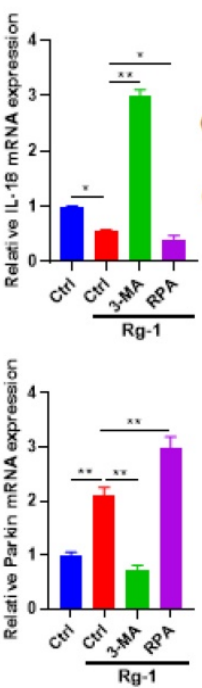

Rg1

B

Ctrl Ctrl 3-MA RPA

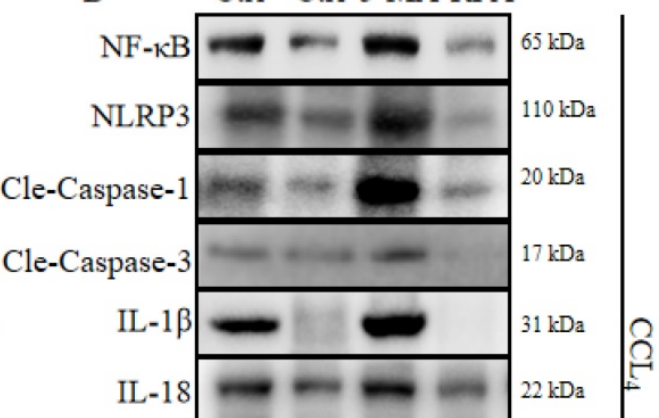

Figure 5 G-Rg 1-promoted autophagy was associated with the NF-KB/NLRP3 inflammasome signaling pathway. (A) The mRNA levels of NF-KB, NLRP3 inflammasome, cleaved caspase 1, cleaved caspase 3, IL-18, IL-1 3 , LC3-II, Beclin-1, PINK1 and Parkin were tested by qRT-PCR. (B) Protein levels of NF-KB, NLRP3 inflammasome, caspase 1, caspase 3, IL-1 $\beta$, IL-18, LC3-I, LC3-II, Beclin-1, PINK1, Parkin were tested by Western blot $(\mathrm{n}=3)$. $* \mathrm{P}<0.05$, $* * \mathrm{P}<0.01, \mathrm{NS}$, no significant.

\section{Discussion}

G-Rg1 exerts strong hepatoprotective properties in the animal models of liver injury, but the protective mechanism needs to be further explored. In this study, we found that G-Rg1 could improve liver function, inhibit inflammatory reaction and hepatocyte apoptosis through promoting autophagic activity in mice with CCl4-induced acute liver injury.

G-Rg1 is the primary pharmacologicallyactive compound in Panax ginseng [23, 24]. The trophic and protective effects of G-Rg1 have been reported in angiogenesis [9], neuroprotection [10, 24], progenitor cell proliferation [11, 12] and inhibition of renal fibrosis [25]. G-Rg1 also possesses hepatoprotective effects in several liver injury models including ischemia/reperfusion injury, lipopolysaccharide/ D-galactosamine-induced acute liver failure, hepatic fibrosis and alcoholic liver disease [26-29]. In our previous study, G-Rg1 could significantly reduce liver damage in a murine model with CCl4-induced acute liver failure through inhibiting TNF-a-induced and caspase-dependent hepatocellular apoptosis [15].

Several trials have reported the effects of G-Rg1 on the autophagy, but they showed different outcomes in certain situations. For example, in the cardiomyocytes hypoxia/reoxygenation model, G-Rg1 could inhibite the autophagy and apoptosis in the H9c2 cardiomyocytes [30]. Another trial have reported that G-Rg1 could improve the survival rate and ameliorated cognitive impairments partially through regulating cerebral inflammation and apoptosis which might possibly via suppressing the non-canonical beclin 1-independent autophagy pathway [31]. In contrast, G-Rg1 was effective to induce the autophagic flux and attenuate serum deprivation-induced apoptosis in Raw264.7 macrophages [32]. The inhibition of apoptosis and promotion of autophagy were also observed in H9c2 cells after G-Rg1 treatment [33].

To the best of our knowledge, this is the first research to explore the effect of G-Rg1 on autophagy in CCl4-induced acute liver injury. Our results showed that the activation of autophagy has important protective effects in CCl4-induced acute liver injury, and G-Rg1 showed the significant capability to promote autophagy and suppress hepatocyte apoptosis in the mice model. Additionally, this research revealed that NLRP3 inflammasome may play an important role in the aggravation of liver function and the elevation of inflammatory responses. In our previous study, G-Rg1 treatment could significantly decrease the expression of NF-kB p65 DNA and NF-kB p65 protein in the mouse model of CCl4-induced acute liver failure [15]. G-Rg1 could protect liver function against hepatic ischemia/ reperfusion injury, CCl4-induced liver injury and hepatic fibrosis via inhibiting NF- $\mathrm{kB}$ activation and 
preventing inflammation [34-38].

NF-KB/NLRP3 inflammasome signaling pathway has participated in the regulation of various diseases. When mesangial cells (MCs) cultured under high- and low-glucose conditions, the lincRNA-Gm4419 might regulate the inflammation, fibrosis and proliferation through NF-KB/NLRP3 inflammasome signaling pathway, which provided new insights into the progression of diabetic nephropathy [39]. NF-kB/NLRP3 inflammasomemediated inflammation had the key role in the dysfunction of human umbilical vein endothelial cell in the high glucose condition [40]. In addition, NF-kB/NLRP3 inflammasomes pathway was associated with the atherosclerotic lesions, and artemisinin might reduce atherosclerotic lesions by suppression of inflammatory reaction via the adenosine $5^{\prime}$-monophosphate activated protein kinase (AMPK)/NF-kB/NLRP3 inflammasomes signalling in macrophages [41].

There were NF-kB-binding sites in the NLRP3 inflammasome promoter, and NF-kB p50 have been reported to be bound to the NLRP3 inflammasome promoter [39]. Furthermore, G-Rg1-triggered autophagy was associated with the reduction in NF-kB and NLRP3 inflammasome. Consistently, the inhibition of G-Rg1-triggered autophagy was associated with the increase in NF-KB and NLRP3 inflammasome. These studies suggested that G-Rg1-triggered autophagy might induce the protective effect in $\mathrm{CCl} 4$-induced acute liver injury through suppressing NF-kB/NLRP3 inflammasome signaling pathway. In turn, NF-kB was known as an important negative regulator of autophagy $[42,43]$.

\section{Conclusion}

G-Rg1 ameliorated acute liver injury via the autophagy, which may be related to NF-KB/NLRP3 inflammasome signaling pathway.

\section{Material and Methods}

\section{Animal experiments}

Male C57BL/ 6 mice (6-8 weeks) were provided by the Laboratory Animal Center of Chongqing Medical University. All of the animals were placed in a specific pathogen-free environment and all surgical procedures and care administered to the animals were approved by the institutional ethic committee. The mice received the intraperitoneal injection of a mixture of carbon tetrachloride $(\mathrm{CCl} 4,50 \%)$ and oil $(50 \%)$ at a dose of $2 \mathrm{ml} / \mathrm{kg}$ body weight. They were scarified after the CCl4 injection for 24 h. $0.2 \mathrm{ml} \mathrm{G}-\mathrm{Rg} 1$ (4 mg/ml, Jicui Biotechnology Co., Ltd., Yunnan, China) was given after the CCl4 injection.
The suppression of autophagy was achieved by the tail vein injection of 3-methyladenine (3-MA, 1 $\mathrm{mg} / \mathrm{kg}$, Sigma) $2 \mathrm{~h}$ before CCl4 exposure, while intraperitoneal injection of autophagy agonist rapamycin (RPA, $1 \mathrm{mg} / \mathrm{kg}$, Sigma) was given $0.5 \mathrm{~h}$ before $\mathrm{CCl} 4$ exposure. Intraperitoneal injections of MCC950 (10 mg/kg, MedChem Express, Shanghai, China) or $0.3 \mathrm{ml}$ ATP (100 Mm, Santa Cruz Biotechnology) was used to inhibit or activate NLRP3 inflammasome.

\section{Enzyme-linked immunosorbent assay (ELISA)}

ELISA kits specific for mouse ALT, AST, TNF- $a$, IL-1 $\beta$ and IL-6 (Cloud-Clone Corp. Wuhan, China) were used to measure their serum levels according to the manufacturer's instructions.

\section{Western blot analysis}

After the designated treatments were performed, liver tissues and cell pellets were lysed with RIPA buffer supplemented with protease inhibitors. Total proteins were separated via $12 \%$ SDS-polyacrylamide gel electrophoresis (PAGE) and transferred to polyvinylidene difluoride (PVDF) membranes. The membranes were incubated overnight with rabbit antibodies against NLRP3 (Zymed, South San Francisco, CA, USA), cleaved caspase 1, cleaved caspase 3 (BD PharMingen, San Diego, California, USA), IL-1 $\beta$ (R\&D Systems, USA), IL-18 (Santa Cruz Biotechnology Inc., Santa Cruz,California, USA), LC3-I, LC3-II (Sigma, USA), NF-kB, Beclin-1, PINK1, Parkin and $\beta$-actin primary antibodies (Cell Signaling Technology, Danvers, MA, USA) at $4^{\circ} \mathrm{C}$. Then, the membranes were treated with a horseradish peroxidase-conjugated goat anti-rabbit secondary antibody (Cell Signaling, CA, USA) and developed with a chemiluminescent substrate (Thermo Fisher Scientific, Rockford, IL, USA). Densitometry analysis was performed using ImageJ software, and the relative levels of protein in each group were normalized to the loading control.

\section{Quantitative real-time polymerase chain reaction (qRT-PCR)}

Total RNA was extracted using TRIzol reagent (Invitrogen, NY, USA) according to the manufacturer's instructions. First-strand cDNA was synthesized from RNA (Superscript III cDNA Synthesis Kit, Invitrogen), and the mRNA levels were estimated by QPCR using a SYBR Green PCR Kit (Invitrogen, NY, USA) with a real-time PCR system (ABI PRISM 7300, MA, USA). The relative quantity of the cycle threshold value was normalized to an internal primer. 


\section{Flow cytometry}

The percentage of autophagy was analyzed using flow cytometry with acridine orange dyeing. Cells were stained with acridine orange $(10 \mathrm{ug} / \mathrm{ml})$ (Invitrogen, Carlsbad, CA, USA) and analyzed using flow cytometry for autophagy. Changes in acridine orange positive population were determined.

$500 \mathrm{ul}$ binding buffer solution was added to the cell pellet and pipette the cell solution to the microtube that covered by black-paper. Then $2 \mathrm{ul}$ Annexin V-FITC, 5 ul Propidium Iodide were added, followed by $5 \mathrm{~min}$ culture at room temperature. Apoptotic cells were then analyzed using flow cytometry.

\section{Fluorescence microscopy}

Apoptotic cells in liver sections were stained by the TUNEL apoptosis detection kit (KeyGEN BioTECH, Nanjing, China). Nuclei were stained with 49,6-diamino-2-phenylindole (DAPI, $1 \mu \mathrm{g} / \mathrm{ml}$ ). All images were obtained with an inverted fluorescence microscope (Nikon Eclipse E800, Tokyo, Japan).

\section{Statistical analysis}

All data are expressed as the mean \pm standard deviation (SD). Each test was performed in triplicate for each sample. One-way analysis of variance (ANOVA) followed by the post hoc LSD test was performed to compare differences among multiple groups. $\mathrm{P}<0.05$ was considered statistically significant. All data were analyzed using SPSS 11.5 software.

\section{Acknowledgements}

\section{Authors' contributions}

Jinqiu Zhao carried out the molecular genetic studies, participated in the sequence alignment and drafted the manuscript. Shujun Zhang revised the manuscript. Bin He conceived of the study, participated in its design and drafted the manuscript. Xiaosong Li revised the manuscript and made some grammar improving and corrected all the typos. All authors read and approved the final manuscript.

\section{Funding}

This work was supported by National Natural Science Foundation of China (NSFC, 81472057, 81871653), the Natural Science Foundation of Chongqing (grant no. cstc2020jcyj-msxmX0159, cstc2020jcyj-msxmX0281 and cstc2020jcyjmsxmX0224), Chongqing Medical Science Project (grant no. 2018MSXM065), Technology Research Project of Chongqing Education Commission (grant no. KJQN201900449) and Chongqing Medical Science
Project (2020FYYF008).

\section{Competing Interests}

The authors have declared that no competing interest exists.

\section{References}

1. Khoury T, Rmeileh AA, Yosha L, Benson AA, Daher S, Mizrahi M. Drug induced liver injury: review with a focus on genetic factors, tissue diagnosis, and treatment options. Journal of clinical and translational hepatology. 2015; 3:

2. Oh IS, Park S-H. Immune-mediated liver injury in hepatitis B virus infection. Immune network. 2015; 15: 191-8.

3. Allard J, Le Guillou D, Begriche K, Fromenty B. Drug-induced liver injury in obesity and nonalcoholic fatty liver disease. Science Direct. 2019; 85:75-107.

4. Åberg F, Helenius-Hietala J, Puukka P, Färkkilä M, Jula A. Interaction between alcohol consumption and metabolic syndrome in predicting severe liver disease in the general population. Hepatology. 2018; 67: 2141-9.

5. Kieslichova E, Frankova S, Protus M, Merta D, Uchytilova E, Fronek J, et al. Acute liver failure due to Amanita phalloides poisoning: therapeutic approach and outcome. Transplantation proceedings: Elsevier; 2018; 50(1): 192-7.

6. Rolando N, Wade J, Davalos M, Wendon J, Philpott-Howard J, Williams R. The systemic inflammatory response syndrome in acute liver failure. Hepatology. 2000; 32: 734-9.

7. Montrief T, Koyfman A, Long B. Acute liver failure: A review for emergency physicians. The American journal of emergency medicine. 2018; 37(2):329-337.

8. Ren $\mathrm{F}$, Zhang L, Zhang X, Shi H, Wen $\mathrm{T}$, Bai L, et al. Inhibition of glycogen synthase kinase $3 \beta$ promotes autophagy to protect mice from acute liver failure mediated by peroxisome proliferator-activated receptor $\alpha$. Cell death \& disease. 2016; 7: e2151.

9. Xie XS, Liu HC, Wang FP, Zhang CL, Zuo C, Deng Y, et al. Ginsenoside Rg1 modulation on thrombospondin-1 and vascular endothelial growth factor expression in early renal fibrogenesis in unilateral obstruction. Phytotherapy research 2010; 24: 1581-7.

10. $\mathrm{Xu} \mathrm{L}, \mathrm{Chen}$ WF, Wong MS. Ginsenoside Rg1 protects dopaminergic neurons in a rat model of Parkinson's disease through the IGF-I receptor signalling pathway. British journal of pharmacology. 2009; 158: 738-48.

11. Shen L-h, Zhang J-t. Ginsenoside Rg1 promotes proliferation of hippocampal progenitor cells. Neurological research. 2004; 26: 422-8.

12. Shi A-w, Wang X-b, Lu F-x, Zhu M-m, Kong X-q, Cao K-j. Ginsenoside Rg1 promotes endothelial progenitor cell migration and proliferation. Acta Pharmacologica Sinica. 2009; 30: 299

13. Wu CF, Bi XL, Yang JY, Zhan JY, Dong YX, Wang JH, et al. Differential effects of ginsenosides on NO and TNF- $\alpha$ production by LPS-activated N9 microglia. International immunopharmacology. 2007; 7: 313-20.

14. Wu F, Zhang S, Kang G. Effects of panax notoginseng saponins on the expression of tumor necrosis factor alpha and secretion phospholipase A2 in rats with liver fibrosis. Zhonghua gan zang bing $\mathrm{za}$ zhi= Zhonghua ganzangbing zazhi= Chinese journal of hepatology. 2003; 11: 51-2.

15. Zhao J, Shi Z, Liu S, Li J, Huang W. Ginsenosides Rg1 from Panax ginseng: a potential therapy for acute liver failure patients? Evidence-Based Complementary and Alternative Medicine. 2014; 2014.

16. Dikic I, Elazar Z. Mechanism and medical implications of mammalian autophagy. Nature reviews Molecular cell biology. 2018; 19: 349.

17. Gatica D, Lahiri V, Klionsky DJ. Cargo recognition and degradation by selective autophagy. Nature cell biology. 2018; 20: 233.

18. Doherty J, Baehrecke EH. Life, death and autophagy. Nature cell biology. 2018; 20: 1110-7.

19. Itakura E, Mizushima N. Characterization of autophagosome formation site by a hierarchical analysis of mammalian Atg proteins. Autophagy. 2010; 6: 764-76.

20. Gump JM, Thorburn A. Autophagy and apoptosis: what is the connection? Trends in cell biology. 2011; 21: 387-92.

21. Mizushima N, Levine B, Cuervo AM, Klionsky DJ. Autophagy fights disease through cellular self-digestion. nature. 2008; 451: 1069.

22. Levine B, Deretic V. Unveiling the roles of autophagy in innate and adaptive immunity. Nature Reviews Immunology. 2007; 7: 767.

23. Chang-Xiao L, Pei-Gen X. Recent advances on ginseng research in China. Journal of ethnopharmacology. 1992; 36: 27-38.

24. Xie W, Zhou P, Sun Y, Meng X, Dai Z, Sun G, et al. Protective effects and target network analysis of ginsenoside $\mathrm{Rg} 1$ in cerebral ischemia and reperfusion injury: A comprehensive overview of experimental studies. Cells. 2018; 7: 270

25. Li S-s, He A-l, Deng Z-y, Liu Q-f. Ginsenoside-Rg1 Protects against Renal Fibrosis by Regulating the Klotho/TGF- $\beta 1 /$ Smad Signaling Pathway in Rats with Obstructive Nephropathy. Biological and Pharmaceutical Bulletin. 2018. 41: 585-91.

26. Li J, Yang C, Zhang S, Liu S, Zhao L, Luo H, et al. Ginsenoside Rg1 inhibits inflammatory responses via modulation of the nuclear factor- $\mathrm{\kappa B}$ pathway and inhibition of inflammasome activation in alcoholic hepatitis. International journal of molecular medicine. 2018; 41: 899-907. 
27. Ning C, Gao X, Wang C, Huo X, Liu Z, Sun H, et al. Protective effects of ginsenoside Rg1 against lipopolysaccharide/D-galactosamine-induced acute liver injury in mice through inhibiting toll-like receptor 4 signaling pathway. International immunopharmacology. 2018; 61: 266-76.

28. Zou Y, Tao T, Tian Y, Zhu J, Cao L, Deng X, et al. Ginsenoside Rg1 improves survival in a murine model of polymicrobial sepsis by suppressing the inflammatory response and apoptosis of lymphocytes. journal of surgical research. 2013; 183: 760-6.

29. Shen L, Zhang J. Ginsenoside Rg1 increases ischemia-induced cell proliferation and survival in the dentate gyrus of adult gerbils. Neuroscience Letters. 2003; 344: 1-4.

30. Zhang Z-L, Fan Y, Liu M-L. Ginsenoside Rg1 inhibits autophagy in H9c2 cardiomyocytes exposed to hypoxia/reoxygenation. Molecular and cellular biochemistry. 2012; 365: 243-50.

31. Li Y, Wang F, Luo Y. Ginsenoside Rg1 protects against sepsis-associated encephalopathy through beclin 1-independent autophagy in mice. Journal of Surgical Research. 2017; 207: 181-9.

32. Yang P, Ling L, Sun W, Yang J, Zhang L, Chang G, et al. Ginsenoside Rg1 inhibits apoptosis by increasing autophagy via the AMPK/mTOR signaling in serum deprivation macrophages. Acta biochimica et biophysica Sinica. 2018; 50: 144-55.

33. Li D, Wang J, Hou J, Fu J, Chang D, Bensoussan A, et al. Ginsenoside Rg1 protects starving $\mathrm{H} 9 \mathrm{c} 2$ cells by dissociation of Bcl-2-Beclin1 complex. BMC complementary and alternative medicine. 2016; 16: 146.

34. Gao Y, Chu S, Li J, Li J, Zhang Z, Xia C, et al. Anti-inflammatory function of ginsenoside Rg1 on alcoholic hepatitis through glucocorticoid receptor related nuclear factor-kappa B pathway. Journal of ethnopharmacology. 2015; 173: 231-40.

35. Tao T, Chen F, Bo L, Xie Q, Yi W, Zou Y, et al. Ginsenoside Rg1 protects mouse liver against ischemia-reperfusion injury through anti-inflammatory and anti-apoptosis properties. journal of surgical research. 2014; 191: 231-8.

36. Zhang X-J, He C, Li P, Su H, Wan J-B. Ginsenoside Rg1, a potential JNK inhibitor, protects against ischemia/reperfusion-induced liver damage. Journal of functional foods. 2015; 15: 580-92.

37. Xin Y, Wei J, Chunhua M, Danhong Y, Jianguo Z, Zongqi C, et al. Protective effects of ginsenoside Rg1 against carbon tetrachloride-induced liver injury in mice through suppression of inflammation. Phytomedicine. 2016; 23: 583-8.

38. Li J-p, Gao Y, Chu S-f, Zhang Z, Xia C-y, Mou Z, et al. Nrf2 pathway activation contributes to anti-fibrosis effects of ginsenoside $\mathrm{Rg} 1$ in a rat model of alcohol-and CCl 4-induced hepatic fibrosis. Acta Pharmacologica Sinica. 2014; 35: 1031.

39. Yi H, Peng R, Zhang L-y, Sun Y, Peng H-m, Liu H-d, et al. LincRNA-Gm4419 knockdown ameliorates NF-KB/NLRP3 inflammasome-mediated inflammation in diabetic nephropathy. Cell death \& disease. 2017; 8: e2583.

40. Cheng J, Liu Q, Hu N, Zheng F, Zhang X, Ni Y, et al. Downregulation of hsa_circ_0068087 ameliorates TLR4/NF-KB/NLRP3 inflammasome-mediated inflammation and endothelial cell dysfunction in high glucose conditioned by sponging miR-197. Gene. 2019; 709: 1-7.

41. Jiang Y, Du H, Liu X, Fu X, Li X, Cao Q. Artemisinin alleviates atherosclerotic lesion by reducing macrophage inflammation via regulation of AMPK/NF-KB/NLRP3 inflammasomes pathway. Journal of drug targeting. 2020; 28(1): 70-79.

42. Djavaheri-Mergny M, Amelotti M, Mathieu J, Besancon F, Bauvy C, Souquere $\mathrm{S}$, et al. NF-kappaB activation represses tumor necrosis factor-alpha-induced autophagy. The Journal of biological chemistry. 2006; 281: 30373-82.

43. Zhu BS, Xing CG, Lin F, Fan XQ, Zhao K, Qin ZH. Blocking NF-kappaB nuclear translocation leads to p53-related autophagy activation and cell apoptosis. World journal of gastroenterology. 2011; 17: 478-87. 